\title{
Routes of transmission of SARS-CoV-2 and behaviours to block it: a summary
}

Robert West ${ }^{1}$, Susan Michie

1 University College London, University of London

Abstract

The article briefly describes the main routes of transmission of the SARS-CoV-2 in the community. It also shows key actions required to prevent this transmission. All these behaviours are important but maintaining physical distance and not touching one's TZone could be particularly important in blocking the final pathways to infection.

\section{Definitions}

Personal protective behaviour

Defined by Robert West et al.

Respiratory viruses such as SARS-CoV-2 have the capability to spread rapidly between people ${ }^{[1]}$. The combination of infectiousness, including when asymptomatic, and severity of the consequent disease means that these viruses can cause very large numbers of deaths, overwhelm health systems and wreak havoc in the global population. Large scale 'lock downs' and quarantine can be used to limit the spread of these viruses but the cost to society is massive. They are necessary because of the difficulty in securing adequate adherence to what may be termed personal protective behaviours. These are behaviours that individuals can enact to protect themselves and others from spread of infection. This article briefly summarises these behaviours. 


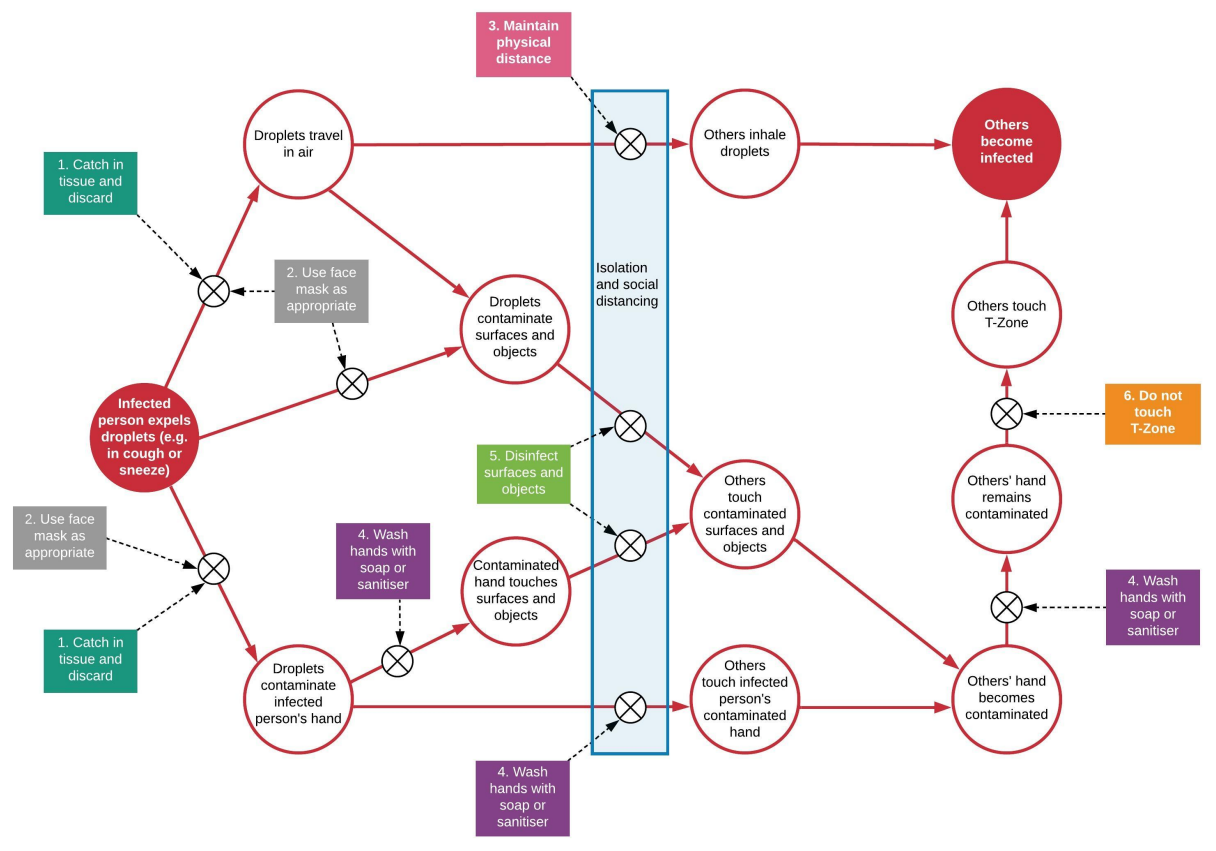

Figure 1: Transmission pathways for SARS-CoV-2 and behaviours needed to block these. The arrows to the left of the blue bar relate to the infected person while those to the right relate to other people who may become infected.

Figure 1 shows the pathways to transmission and the actions required to block it. The vertical blue bar represents the upstream measures (isolation and social distancing) that aim to create physical distance between people and between people and potentially contaminated objects and surfaces. Isolation and social distancing are being defined in varying ways but in general isolation means maintaining a physical barrier or distance between a person (e.g. someone with extreme vulnerability) and all other people as well as potentially contaminated objects; social distancing means staying at home with one's family (if one has one) and only going out for essential journeys. To achieve social distancing, governments have closed shops and businesses and banned gatherings and movement outside of one's immediate environment. These measures are having catastrophic effects on many people's lives, the global economy and societal infrastructure.

The need for such draconian measures arises because of the difficulty getting people to adhere to the downstream personal protective behaviours required to block transmission. Enacting these behaviours with sufficient rigour is not just a matter of motivation but also requires the capability (e.g. understanding and skill) and opportunity (e.g. resources, circumstances and physical space). 


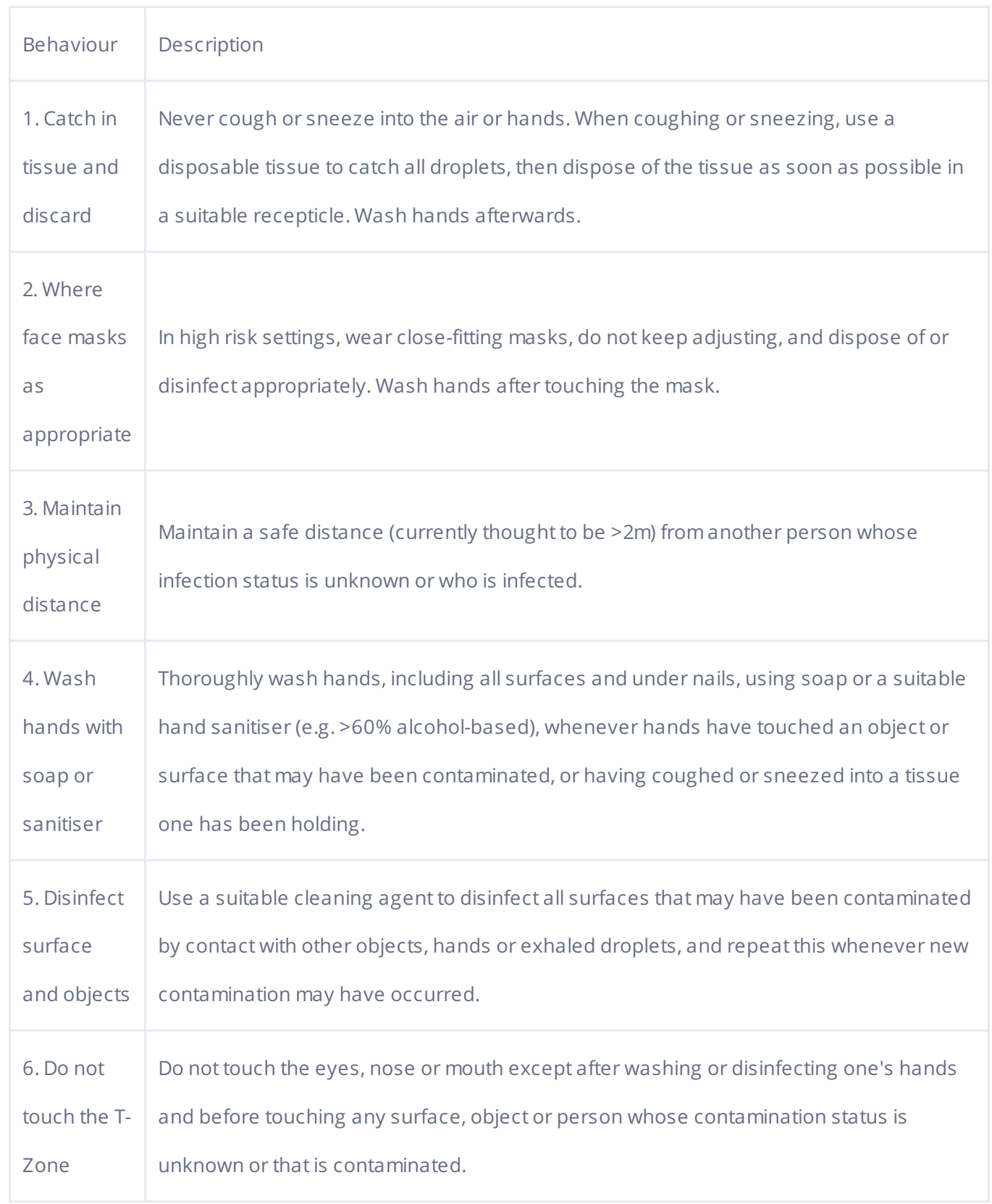

Table 1: Personal protective behaviours relating to SARS-CoV-2

Wearing face masks in community settings is currently a matter of discussion because if used inappropriately there is a risk they may increase virus transmission by acting as fomites ${ }^{[2]}$. This may change as new information becomes available. It may be noted that there are two behaviours in particular that could block the final pathway: keeping physical distance and not touching one's T-Zone (eyes nose and mouth). The physical distancing is important but would not be enough on its own. Not touching the T-Zone is crucial because the virus appears to be able to live on surface for up to several days in some cases and surfaces can easily become re-contaminated after being washed, as can hands ${ }^{[3]}$

References 
1. ^ Mary A Lake. (2020). What we know so far: COVID-19 current clinical knowledge and research. Clin Med, vol. 20 (2), 124-127. doi:10.7861/clinmed.2019-coron.

2. `Shuo Feng, Chen Shen, NanXia, Wei Song, Mengzhen Fan, BenjaminJ Cowling. (2020). Rational use of face masks in the COVID-19 pandemic. The Lancet Respiratory Medicine. doi:10.1016/s2213-2600(20)30134-x.

3. ^Neeltje van Doremalen, Trenton Bushmaker, Dylan H. Morris, Myndi G. Holbrook, Amandine Gamble, Brandi N. Williamson. (2020). Aerosol and Surface Stability of SARSCoV-2 as Compared with SARS-CoV-1. N Eng/J Med. doi:10.1056/nejmc2004973. 